\title{
Presentation and Prognosis of Incomplete Resection in Thymic Carcinoma
}

\author{
Rahul K. Bhardwaj
}

\begin{abstract}
We present a case of a 65-year-old African American female who presented with a chief complaint of shortness of breath. Computed tomography of the chest revealed an anterior mediastinal mass. Biopsy revealed a thymic carcinoma. Histopathology revealed the rare variant of basaloid subtype of thymic carcinoma. Our case demonstrates the various modalities of treatment in such type of cancer and the poor prognosis associated with it when other structures are involved.
\end{abstract}

Keywords: Thymus cancer; Thymic cancer; Incomplete resection; Basaloid subtype

\section{Introduction}

Thymic cancer is among one of the rarest malignancies, accounting for less than $1 \%$ of all neoplasms [1]. A rare variant is the basaloid subtype which has approximately 10 reported cases in literature [2]. Treatment of such a malignancy is mainly aimed at complete resection, incomplete resection, or a combination of chemotherapy, radiotherapy and resection.

\section{Case Report}

A 65-year-old woman who was recently discharged for treatment of pneumonia presented with shortness of breath. Initial chest X-ray revealed a marked elevation of the right hemidiaphragm vs. subpulmonic effusion. Patient was started on antibiotics and placed on continuous BiPAP. A computed tomography $(\mathrm{CT})$ of chest without contrast was obtained to estimate the effusion quantity which revealed an anterior mediastinal mass measuring approximately $3.6 \times 4.5 \times 5.8 \mathrm{~cm}$. The patient had undergone a biopsy, which provided an inconclusive dif-

Manuscript accepted for publication March 16, 2016

Department of Medicine, Mount Sinai Hospital, 1500 S California, Chicago, IL 60608, USA. Email: rahul.bhardwaj@sinai.org

doi: http://dx.doi.org/10.14740/jmc2446w ferential consisting of thymoma (type B), thymic carcinoma and lung squamous cell carcinoma. A repeat CT scan with contrast showed the anterior mediastinal mass measuring 4.2 $\times 4.9 \times 5.2 \mathrm{~cm}$. CT surgery performed a median sternotomy and was able to incompletely ( $85 \%$ ) remove the mediastinal mass. The resection was limited due to the mass encasing right phrenic nerve and the pulmonary vein. The mass was fixed to the pericardium and involved the right upper lobe of the lung (Fig. 1, 2). Biopsy results of the resected mass showed thymic carcinoma, basaloid carcinoma subtype staging T4NxMx (Fig. 3). Postoperatively, the patient had numerous complications such as requiring vasopressor support, going into A.fib with RVR requiring amiodarone, and having a cardiac arrest which was thought to be due to hypoxia or phrenic nerve paralysis. Considering the patient's poor prognosis, her family decided for full withdrawal of support after which patient passed away.

\section{Discussion}

This case illustrates the poor prognosis observed in patients with incomplete resection of basaloid subtype of thymic cancer when other structures are involved. It is important to consider the high risk with minimal benefit if complete resection is unlikely [3]. With our patient, it was difficult to determine

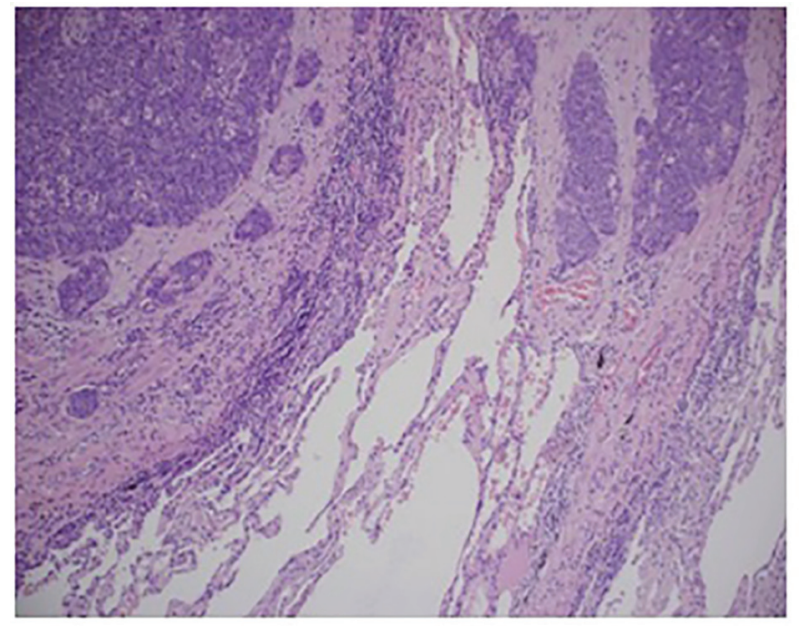

Figure 1. Tumor invading the lung parenchyma $(\times 10)$. 


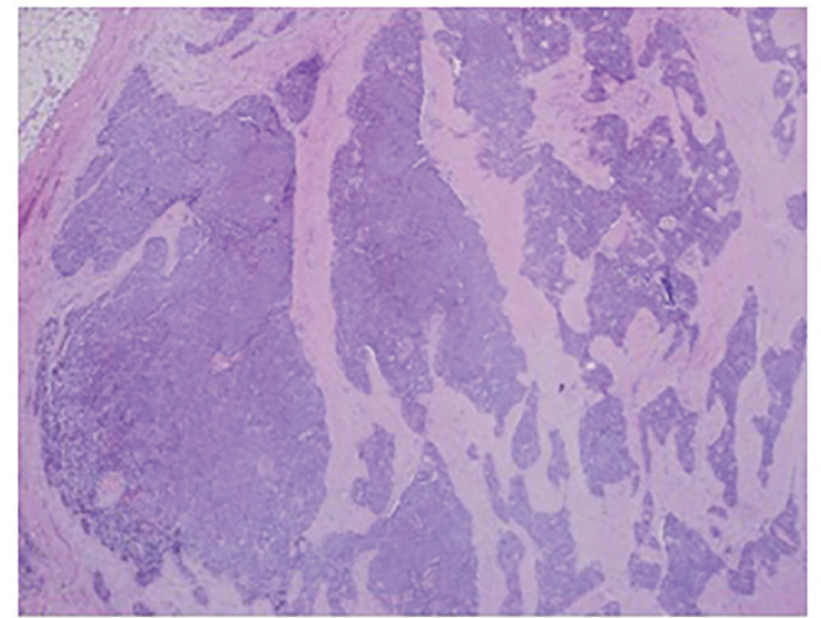

Figure 2. Infiltrating carcinoma in fibrotic background $(\times 4)$.

if complete resection was possible. In such cases, deciding between incomplete resection and no surgical intervention should be considered.

\section{References}

1. Travis WDBE, Muller-Hermelink HK, Harris CC. WHO histological classification of tumors of the lung. In: World Health Organization Classification of Tumours Pathology

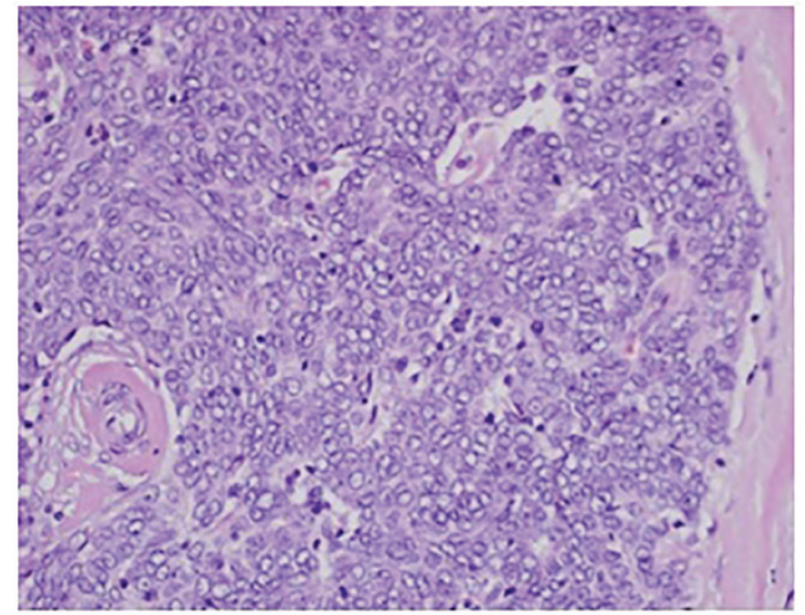

Figure 3. Neoplastic basaloid epithelial cells with mitotic figures $(\times 40)$.

and Genetics of Tumours of the Lung, Pleura, Thymus and Heart. Lyon: IARC Press; 2004.

2. Sakakura N, Tateyama H, Usami N, Yokoi K. Thymic basaloid carcinoma with pleural dissemination that developed after a curative resection: report of a case. Surg Today. 2010;40(11):1073-1078.

3. Ogawa K, Toita T, Uno T, Fuwa N, Kakinohana Y, Kamata M, Koja K, et al. Treatment and prognosis of thymic carcinoma: a retrospective analysis of 40 cases. Cancer. 2002;94(12):3115-3119. 